\title{
ABOUT THE PECULIARITIES OF THE POLARIZATION APPROACH TO THE MEASURING OF COHERENCE PROPERTIES OF OPTICAL FIELD
}

Claudia Yu. Zenkova ${ }^{1}$, Oleg V. Angelsky ${ }^{2}$, Nina V. Gorodyns'ka ${ }^{2}$

Chernivtsi National University, ${ }^{1}$ Optics and Spectroscopy Department, ${ }^{2}$ Department of Correlation Optics 2, Kotsyubinsky Str., Chernivtsi 58012, Ukraine

\begin{abstract}
The mathematical estimation of the degree of correlation of optical fields in the near field has been analyzed. The need for taking into account the modulation of polarization and the modulation of intensity in measuring the visibility of the interference pattern has been demonstrated.
\end{abstract}

Keywords: polarization, correlation parameters, coherence.

\section{INTRODUCTION}

A steady growing interest to the study of interconnections among polarization and correlation parameters of a field for describing the coherence properties of optical fields has been observed during the last years ${ }^{1,2}$. There are two reasons for this:

- on the one hand, it is necessary to elucidate in detail differences of the classical approaches in the theory of optical coherence (Glauber, Wolf) ${ }^{1-4}$;

- on the other hand, the growth of the number of studied objects inhomogeneous from the viewpoint of the polarization state, due to the involvement of biological tissues, calls for extending the list of statistical polarization parameters which can be used as reliable parameters for the diagnosis of such objects, in part in the problems of optical coherent polarization tomography ${ }^{5}$.

Nowadays, the analysis of these problems shows that in papers ${ }^{6,7}$ the parameter characterizing the degree of mutual polarization in different points of inhomogeneously polarized optical fields has been introduced and accepted. This parameter is invariant to the transformations of the coordinate system and, as a consequence, does not depend on the choice of the state of polarization of the probing beam and determines according to the following expression ${ }^{6}$

$\gamma_{W}^{2}\left(\vec{r}_{1}, \vec{r}_{2}, \tau\right)=\frac{\operatorname{tr}\left[W\left(\vec{r}_{1}, \vec{r}_{2}, \tau\right) W\left(\vec{r}_{2}, \vec{r}_{1},-\tau\right)\right]}{\operatorname{tr} W\left(\vec{r}_{1}, \vec{r}_{1}, 0\right) \operatorname{tr} W\left(\vec{r}_{2}, \vec{r}_{2}, 0\right)}=\frac{\sum_{i, j}\left|W_{i, j}\left(\vec{r}_{1}, \vec{r}_{2}, \tau\right)\right|^{2}}{\sum_{i, j} W_{i i}\left(\vec{r}_{1}, \vec{r}_{1}, 0\right) W_{j j}\left(\vec{r}_{2}, \vec{r}_{2}, 0\right)}$,

where $\mathrm{W}\left(\overrightarrow{\mathrm{r}}_{1}, \overrightarrow{\mathrm{r}}_{2}, \tau\right)$ - is the $3 \times 3$ mutual coherence matrix, defined by

$W\left(\vec{r}_{1}, \vec{r}_{2}, \tau\right)=<\vec{E}_{i}\left(\vec{r}_{1}, \tau\right) \vec{E}_{j}{ }^{*}\left(\vec{r}_{2}, \tau\right)>,(\mathrm{i}=\mathrm{x}, \mathrm{y}, \mathrm{z})$

where $\mathrm{i}, \mathrm{j}=\mathrm{x}, \mathrm{y}, \mathrm{z}$, and we have made use of the Hermiticity relation $W_{i j}^{*}\left(\vec{r}_{1}, \vec{r}_{2}, \tau\right)=W_{j i}\left(\vec{r}_{2}, \vec{r}_{1},-\tau\right)$ satisfied by the electric coherence-matrix elements. It is well-known, that the coherence properties at certain moment of time between spatiotemporal points $\vec{r}_{1}$ and $\vec{r}_{2}$ of the complex random vectors $\vec{E}\left(\vec{r}_{1}, \tau\right)$ and $\vec{E}\left(\vec{r}_{2}, \tau\right)$ can be represented by the mutual coherence matrix $\mathrm{W}\left(\vec{r}_{1}, \vec{r}_{2}, \tau\right)$, defined by the Exp. (2). The quantity $\gamma_{\mathrm{W}}$ was called as the degree of coherence for electromagnetic fields ${ }^{6}$, or the degree of mutual polarization ${ }^{7}$.

One can note that the standard coherence matrix corresponds to the case $\vec{r}_{1}=\vec{r}_{2}=\vec{r}$ at the fixed moment of time. The standard coherence matrix will be called, the polarization matrix, which will thus correspond to $\Gamma(\vec{r})=<\vec{E}(\vec{r}) \vec{E}^{*}(\vec{r})>$.

Since the degree of mutual polarization contains information about the correlation that exist between the components of the electric field at a pair of points, it might expect that there exist a connection between 
$\gamma_{\mathrm{W}}$ and the degree of polarization that characterizes correlations in a single point. It has been once more theoretically substantiated that the degree of polarization characterizes the level of disorder in each beam, viz. the level of decorrelation of the field components of one beam, and corresponds to the maximum of the autocorrelation function of this beam ${ }^{8}$.

In its turn, the intrinsic coherence introduced in the paper ${ }^{1}$ characterizes the degree of disorder between interacting beams in different points of a field and at different instants. Henceforth, for the quantitative characteristics of the intrinsic coherence we will use the term degree of cross-correlation of the fields, regarding these terms to be synonyms.

Really a normalized mutual coherence matrix $M\left(\vec{r}_{1}, \vec{r}_{2}, t_{1}, t_{2}\right)=\Gamma^{-1 / 2}\left(\vec{r}_{2}, t_{2}\right) W\left(\vec{r}_{1}, \vec{r}_{2}, t_{1}, t_{2}\right) \Gamma^{-1 / 2}\left(\vec{r}_{1}, t_{1}\right)$,

whose singular values $\mu_{s}\left(\vec{r}_{1}, \vec{r}_{2}, t_{1}, t_{2}\right), \mu_{l}\left(\vec{r}_{1}, \vec{r}_{2}, t_{1}, t_{2}\right)$ determine the intrinsic degrees of coherence $e^{8,9}$. From mathematical point of view, one uses the property that any matrix can be decomposed in singular values $\mu_{s}\left(\vec{r}_{1}, \vec{r}_{2}, t_{1}, t_{2}\right), \mu_{l}\left(\vec{r}_{1}, \vec{r}_{2}, t_{1}, t_{2}\right)$, such as $M\left(\vec{r}_{1}, \vec{r}_{2}, t_{1}, t_{2}\right)=N_{2}^{*} D\left(\vec{r}_{1}, \vec{r}_{2}, t_{1}, t_{2}\right) N_{1}$,

where $D\left(\vec{r}_{1}, \vec{r}_{2}, t_{1}, t_{2}\right)=\left[\begin{array}{cc}\mu_{s}\left(\vec{r}_{1}, \vec{r}_{2}, t_{1}, t_{2}\right) & 0 \\ 0 & \mu_{l}\left(\vec{r}_{1}, \vec{r}_{2}, t_{1}, t_{2}\right)\end{array}\right]$,

$\mathrm{N}_{1}$ and $\mathrm{N}_{2}$ are unitary matrices and where $\mu_{s}\left(\vec{r}_{1}, \vec{r}_{2}, t_{1}, t_{2}\right) \geq \mu_{l}\left(\vec{r}_{1}, \vec{r}_{2}, t_{1}, t_{2}\right) \geq 0$. The matrix $D\left(\vec{r}_{1}, \vec{r}_{2}, t_{1}, t_{2}\right)$ corresponds to the normalized mutual coherence matrix of totally depolarized light described in the basis of the singular value decomposition of $M\left(\vec{r}_{1}, \vec{r}_{2}, t_{1}, t_{2}\right)$.

Let us introduce the term the degree of cross-correlation of the field. Under this term, in our approach, we understand the correlation of collinear components of the interacting beams, for example, $E_{x 1}\left(\vec{r}_{2}, t_{2}\right), E_{x 2}\left(\vec{r}_{1}, t_{1}\right), E_{z 1}\left(\vec{r}_{2}, t_{2}\right), E_{z 2}\left(\vec{r}_{1}, t_{1}\right)$, which in there turn determine the degrees of the intrinsic coherence $\mu_{s}\left(\vec{r}_{1}, \vec{r}_{2}, t_{1}, t_{2}\right), \mu_{l}\left(\vec{r}_{1}, \vec{r}_{2}, t_{1}, t_{2}\right)$, according to Exp. (4) - (6). In this sense the conceptions of the intrinsic coherence and the cross correlation are identical. We take into consideration the longitudinal field component (z-component) in order to be able to demonstrate the contribution of the polarization modulation into the degrees of intrinsic coherence. In our case of the interference of two orthogonally polarized waves the normalized matrix of mutual coherence will be put down as

$M\left(\vec{r}_{1}, \vec{r}_{2}, t_{1}, t_{2}\right)=\left[\begin{array}{cc}\eta_{x x}\left(\vec{r}_{1}, \vec{r}_{2}, t_{1}, t_{2}\right) & 0 \\ 0 & \eta_{z z}\left(\vec{r}_{1}, \vec{r}_{2}, t_{1}, t_{2}\right)\end{array}\right]$,

where $\eta_{i, j}\left(\vec{r}_{1}, \vec{r}_{2}, t_{1}, t_{2}\right)=\frac{<E_{i}\left(\vec{r}_{2}, t_{2}\right) E_{j}^{*}\left(\vec{r}_{1}, t_{1}\right)>}{\sqrt{l_{i}\left(\vec{r}_{2}, t_{2}\right) l_{j}\left(\vec{r}_{1}, t_{1}\right)}}, i, j=x, z$.

Here $\mu_{s}\left(\vec{r}_{1}, \vec{r}_{2}, t_{1}, t_{2}\right)=\eta_{x x}\left(\vec{r}_{1}, \vec{r}_{2}, t_{1}, t_{2}\right)$ and it describes the degree of the coherence between $x-$ components of the two initial waves. Correspondingly $\mu_{l}\left(\vec{r}_{1}, \vec{r}_{2}, t_{1}, t_{2}\right)=\eta_{z z}\left(\vec{r}_{1}, \vec{r}_{2}, t_{1}, t_{2}\right)$ and describes the degree of coherence between z-components. The maximum values of the parameters $\mu_{s}$ and $\mu_{l}$ are unit and correspond to completely correlation of $x$ - and $z$-components. If the both intrinsic degrees of coherence are equal to one, there exists a polarization modulation of the field that can lead to a unit modulus of the visibility of the interference pattern.

It has been also shown ${ }^{9}$ that the degrees of polarization and the intrinsic degrees of coherence between the beams appear to be additive contributions to the entropy, with the same functional dependence. At the same time, the question remains opened: in what way one can experimentally estimate the intrinsic coherence in real optical fields, where the correlation of the fields can manifest both in the spatial modulation of intensity due to interference and in the polarization modulation of the resulting field ${ }^{10,11}$. In part, it can manifest in the near field of diffraction, where the contribution from the longitudinal component of the field is essential and, consequently, the polarization modulation of the field is also rather pronounced. It seems that taking into account the contribution of this component to the correlation of the fields is of importance in the problems of microoptics and near field optics ${ }^{12}$. 


\section{STATEMENT OF THE PROBLEM}

In the general case, the intrinsic coherence must be estimated by measuring both the visibility of the resulting distribution and the deepness of modulation, i.e. the degree of polarization of the field. The sum of these two normalized parameters must be equal to unity. It seems that the additive properties of correlations manifests itself just in this manner. In other words, the degree of polarization and the degree of coherence of the field determined by the visibility of the interference pattern are mutually complementary measures of the cross-correlation of two fields.

The feasibilities of the estimating of the degree of two fields correlation are presented. They are depended on the experimental models:

- interference of identically polarized beams (scalar approximation), when the degree of cross-correlation is estimated by the visibility of the recorded interference pattern;

- superposition of orthogonally polarized interacting beams, when the degree of cross-correlation is estimated by the deepness of polarization state (SOP) modulation, viz. the degree of polarization of the resulting field;

- superposition of the beams whose states of polarization (SOPs) are different but not orthogonal, when for the estimation of for estimating the degree of cross-correlation it is necessary to measure both the visibility of the interference pattern and deepness of modulation of the polarization distribution, i.e. the degree of polarization.

A violation of the condition of rigorous orthogonality of oscillations in the interacting linearly polarized waves will manifest itself in a periodical spatial modulation of intensity. The reason is the change of visibilities of the partial interference patterns shifted by a half-period, Fig. 1(a), that is in its turn caused by different amplitude ratios of the interfering $x-$ and $z$ - components of the corresponding decomposition, Fig. 1(b). At the registration plane one can analyze the intensity modulation and the polarization modulation which yet must be revealed. As a matter of fact, the spatial polarization modulation is realized in the plane coinciding with the plane of the figure. It is expediently to use here the interference principle, namely, to introduce perpendicularly to the registration plane the third plane coherent probing beam which is linearly polarized at the figure plane. One can reliably predict that the interference of such three waves will result in changing visibility (deepness of the intensity modulation) of the interference pattern caused by the in-pair interaction of the collinear components of the two initial waves. To simplify the experiment, the convergence angle of these two initial waves could be chosen in such a manner that the period of the resulting interference pattern would be observed visually using a microscope, and the resulting distribution would be simply registered holographically. Usage of the reference wave enables only to illustrate the presence in the field of the spatial polarization modulation (together with the intensity modulation caused by the interference of the collinear field components). In this case, we only reveal additional changing of the visibility of the resulting pattern of modulation caused by interference of the collinear components of the SOP distribution and the reference wave. A quantitative estimation of the degree of correlation contained in the polarization modulation of a field, however, can be obtained only by making polarization measurements of the degree of mutual polarization of the field. In other words, the quantitative estimation of the intrinsic coherence requires an experimental estimation of the correlation of the four components of the superimposing beams.

Here, to provide correctness to the experiment, it is necessary to compare visually or by measurement two situations:

- when the reference wave is "imposed" on the homogeneously polarized distribution, and

- when the same wave is "imposed" on the modulated in polarization distribution. The experimental arrangement is shown in Fig. 2. CCD camera is used for the registration of the obtained interference patterns. The angle of convergence of the plane object beams is $10^{\circ}$, and the angle of each of the plane object beams with the reference beam is $5^{0}$. The intensities of the object beams were equal to each other, and intensity of the reference beam was changed. The states of polarization of the superimposed beams were also determined. The experimental results are shown in the form of interferograms obtained in various polarization situations. It is seen from the photos shown in Fig. 3(a) that the interference of two plane object waves which are linearly polarized in the plane of the figure results in the interference pattern with the period corresponding to the angle of convergence of the two beams, and the visibility is determined by the ratio of the $x-$ and $z$ - components of the decomposition shown in Fig. 1.

The use of the third linearly polarized beam with the direction of oscillation of the electrical vector perpendicular to the figure plane does not result in any changes in the structure (period) of an 
interference pattern. Only the visibility of the pattern is changed due to changing the level of background, see Fig. 3(b). If the state of polarization of the reference beam is linear and the electrical vector lies in the figure plane, then the structure (period) of the registered interference pattern is changed, as a rule, it is doubled, Fig. 3(c). The doubling of the period of the interference pattern is the most pronounced in the situation in which the intensity of the reference beam exceeds the intensity of the object beams. Such doubling of a period is of pure polarization nature. If intensity of the reference beam is spatially no uniform, one can observe the mechanism of the period doubling of the interference distribution - see the picked out fragment in Fig. 3(d). Thus, the contribution of the polarization component to the correlation of optical fields has been shown. The differences in the structure of the observed interference patterns are the most pronounced in the situation of a three-beam interference, when the intensity of the reference beam is higher than the intensities of the two object beams.

a)

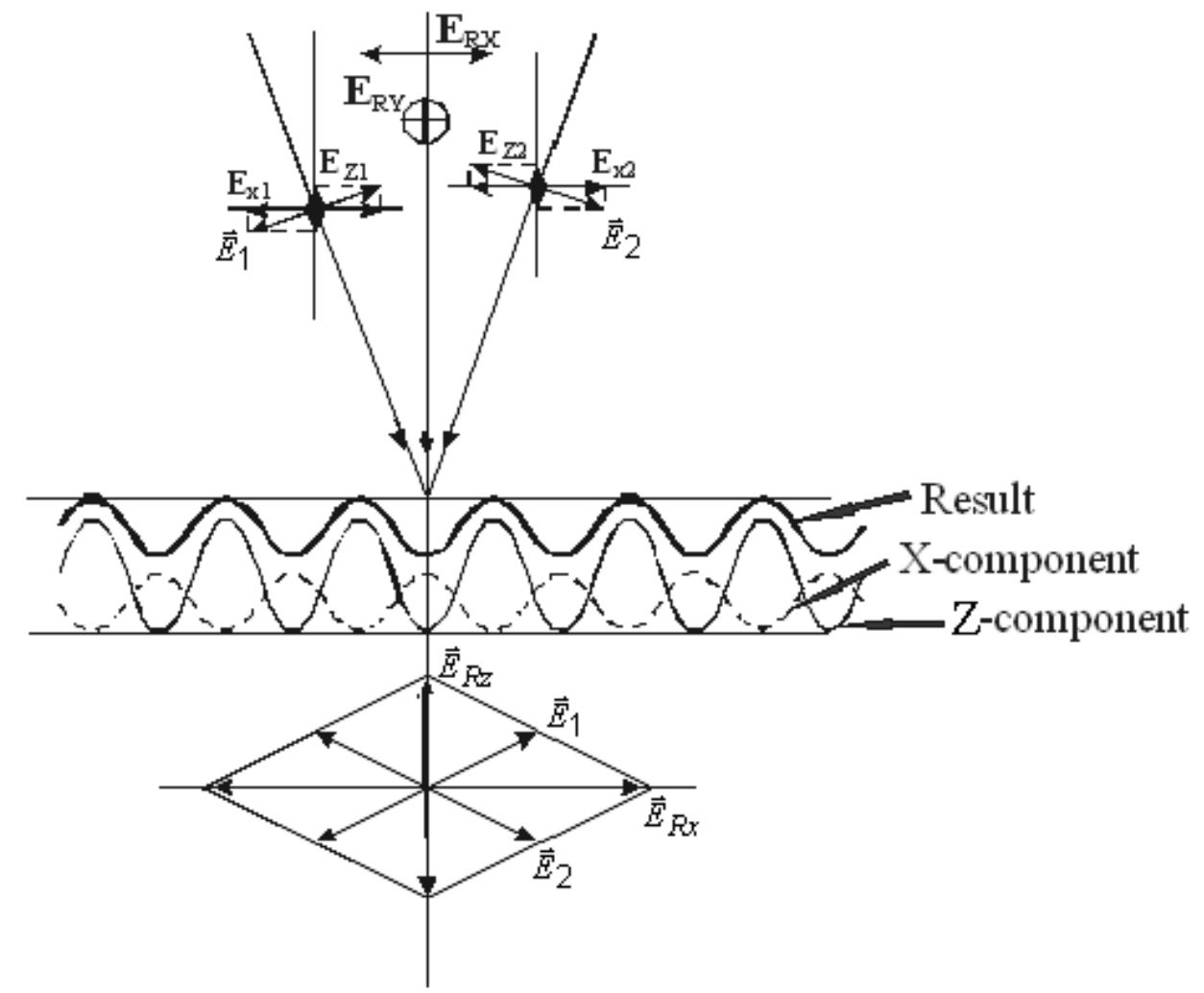

Fig. 1. The result of the interference of two linearly polarized waves $E_{1}$ and $E_{2}$, which manifests itself in:

a) the intensity modulation (Fig. a), which is a result of the in-pair interaction of the $E_{x 1}, E_{x 2}$ and $E_{z 1}, E_{z 2}$ collinear components of the vectors $\vec{E}_{1}$ and $\vec{E}_{2}$ decomposition,

b) the polarization modulation, which can change from the linear with the resulting vector $\vec{E}_{R x}$ to the linear with the resulting vector $\vec{E}_{R z}$ (Fig. b) through the corresponding elliptic polarizations.

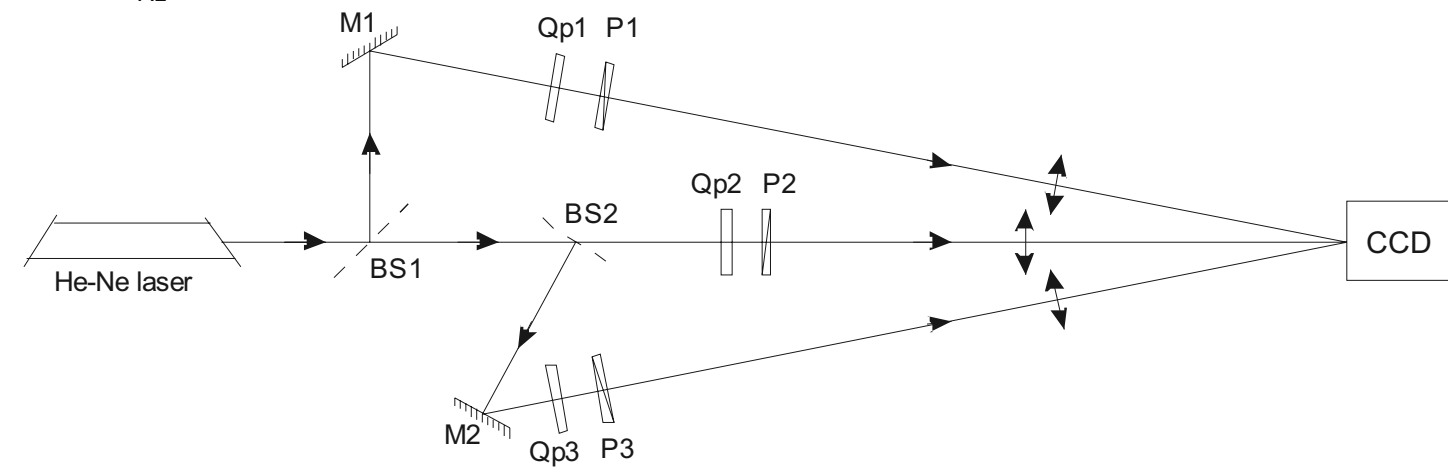

Fig. 2. Optical arrangement of the experiment: BS1, BS2 - beam splitters; M1 and M2 - mirrors; Qp1, Qp2 and Qp3 - quarter-wave plates; P1 - P3 - polarizers; CCD - CCD camera. 


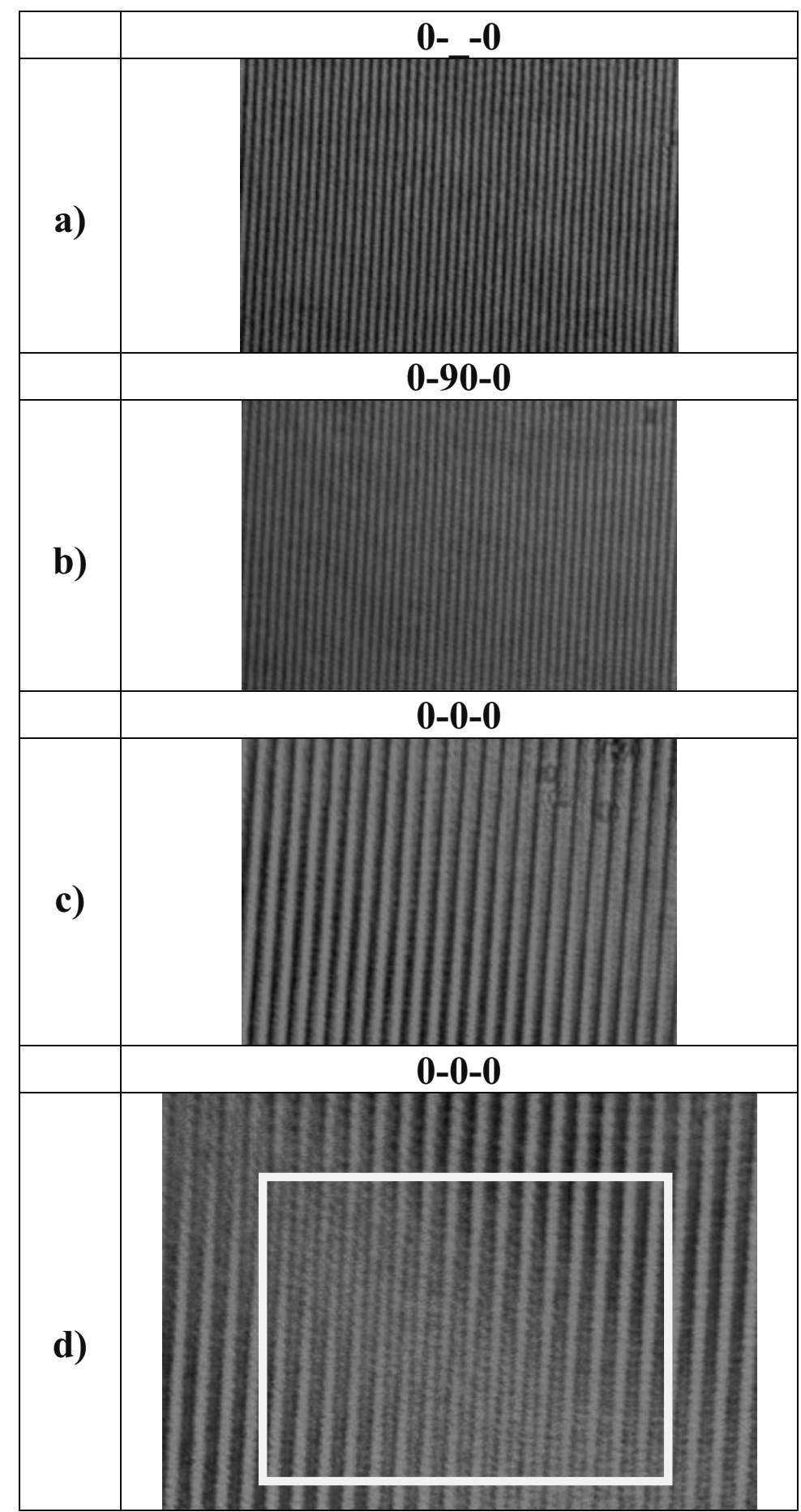

CONCLUSIONS
Fig. 3. a-d. Images of two resulting interferograms:

(a) for two object waves with the plane of polarization in the figure plane;

(b) for two object waves with the plane of polarization in the figure plane and the reference wave with the plane of polarization perpendicular to the figure plane;

(c) for two object waves and the reference wave with the plane of polarization in the figure plane;

(d) the result of doubling the period of an interference pattern for interference of three beams with polarization in the figure plane.
One can certainly assert that only in the pattern of the interference distribution for three beams polarized in the incidence plane one can observe elements, which can be classified as bending or, perhaps, even bifurcations of the interference fringes, see Fig. 3(d). This is the unbiased diagnostic sign of the presence of the polarization singularities ${ }^{14}$ in the field and shows the contribution of the polarization component in the formation of the structure of the interference distribution and, as a consequence, of a field. The estimations of the visibility of the obtained interference distributions, both experimentally and by computer simulation, support this conclusion.

Thus, the contribution of the polarization component in the correlation of the vector optical fields has been substantiated and experimentally illustrated. Possible techniques for the experimental selection of the contribution of the spatial polarization modulation of a field in the general pattern of the distribution of both amplitude and phase parameters and polarization parameters of the field have been considered. It has been noted that the degree of mutual polarization of a field, viz. the parameter which by its sense can be referred to as the generalized visibility of a field, is the optimal statistical parameter providing an estimation of the correlation of the complex spatially statistically inhomogeneous fields. 


\section{REFERENCES}

1. P.Refregier, A. Roueff, "Intrinsic Coherence: A new concept in Polarization and Coherence Theory", OPN, 30-35 (2007).

2. E.Wolf, "Unified theory of coherence and polarization of random electromagnetic beams", Phys. Lett. A 312, 263-267 (2003).

3. R.J.Glauber, "The quantum theory of optical coherence", Phys. Rev. 130, 2529-2539 (1963).

4. L.Mandel, E.Wolf, Optical Coherence and Quantum Optics (Cambridge University Press, 1995).

5. J.F. de Boer, T.E. Milner, "Review of polarization sensitive optical coherence tomography and Stokes vector determination", J.Biomed.Opt. 7, 359-371 (2002).

6. J.Tervo, T.Setala, A.T.Friberg, "Degree of coherence for electromagnetic fields", Opt.Express 11, 10, 1137-1143 (2003).

7. J. Ellis, A. Dogariu, "Complex degree of mutual polarization", Opt.Lett 29, 6, 536-538 (2004).

8. P. Refregier, F. Goudail, "Invariant degrees of coherence of partially polarized light", Opt. Express 13, $16,6051-6060$ (2005).

9. P. Refregier, J. Morio, "Shannon entropy of partially polarized and partially coherent light with Gaussian fluctuations", J.Opt.Soc.A 23, 12, 3036-3044 (2006).

10. T. Tudor, "Waves. Amplitude waves. Intensity waves", Journal of Optics-Paris (Nouv. Rev. Opt.) 22, 6, 291-296 (1991).

11. T. Tudor, "Polarization waves as observable phenomena", J. Opt. Soc. Am. A 14, 8, 2013-2020 (1997).

12. A.Apostol, D.Dogariu, "Non-Gaussian statistics of optical near-fields", Phys.Rev. E 72, 025602, (2005).

13. O.V.Angelsky, N.N. Dominikov, P.P. Maksimyak, T.Tudor, "Experimental revealing of polarization waves", Appl. Opt. 38, 14, 3112-3117 (1999).

14. O.V.Angelsky, I.I.Mokhun, A.I.Mokhun, M.S.Soskin "Interferometric methods in diagnostics of polarization singularities", Physical Review E, 65, 036602 (2002). 